\title{
Impact of the COVID-19 Pandemic on Frailty in Older Adults
}

\author{
(1) Burak Mete, (1) Ferdi Tanır, (1) Hakan Demirhindi, (1) Ayşe İnaltekin, (1) Ceren Kanat \\ Çukurova University Faculty of Medicine, Department of Public Health, Adana, Turkey
}

\begin{abstract}
Objective: This study examines the effects of the Coronavirus disease-2019 (COVID-19) pandemic on frailty in a group of older adults at the end of the first year of the pandemic.

Materials and Methods: The cross-sectional study was conducted at the end of the first year of the pandemic. Our study included 394 older adults who were contacted at primary care health centers. The FRAIL scale, the coronavirus fear scale and the scale of adjustment to measures in respiratory disease outbreaks in the Elderly were used.

Results: The average age of the 394 individuals who participated was $70.38 \pm 5.68$ years. Overall, $33 \%$ of the individuals have been infected with COVID-19. It was found that the prefrail and frail older populations increased by $2.7 \%$ and $13.8 \%$, respectively, in the first year of the COVID-19 pandemic. The pandemic was found to have a moderate effect on the frailty scores. The risk of frailty was found to be 2 [odds ratio $(O R)=2.04$, confidence interval $(\mathrm{CI})(95 \%)=1.28-3.23]$ times higher in individuals that tested positive for COVID-19. The fear of coronavirus increased the risk of frailty by 1.08 times [OR=1.08, $\mathrm{Cl}(95 \%)=1.03-1.13]$. The risk of frailty was reduced by $1.03[\mathrm{OR}=0.96, \mathrm{Cl}(95 \%)=0.94-0.99]$ times in the older adults who complied with the precautions.
\end{abstract}

Conclusion: COVID-19 and the fear of COVID-19 it causes increase the risk of frailty among the older adults. Compliance with the recommended measures reduces the risk of frailty.

Keywords: Aged, COVID-19, frail elderly, pandemics

\section{Introduction}

Turkey confirmed its first case of Coronavirus disease-2019 (COVID-19) on March 11, 2020. Since then, six million people have tested positive for COVID-19, and 53,000 people have died. COVID-19 is highly heterogeneous, with some patients being asymptomatic to others presenting mild to severe symptoms that can lead to death. Factors including age, sex, and comorbid conditions are key determinants of the disease severity and progression. Older age is a prominent risk factor for severe disease and death from COVID-19 (1). There is overwhelming evidence from around the world suggests that age itself is the most significant risk factor for severe COVID-19 disease (2). Early data from China demonstrated that the case fatality ratio (CFR) of COVID-19 increases with age, from $0.4 \%$ or lower in patients aged in their 40 s or younger to $1.3 \%, 3.6 \%, 8 \%$, and $14.8 \%$ in people in their 50s, 60s, 70s, and 80s or older, respectively.
The overall CFR was $2.3 \%$ (3). In comparison, the overall CFR was approximately $2.8 \%$ worldwide and $2.7 \%$ in the United States as of October 19, 2020. The rising number of older adults worldwide, coupled with the unique socio-economic context, ongoing healthcare reform, and the growing development of geriatrics, creates significant challenges in combating the spread of COVID-19 (4). There is a consensus that older adults are one of the most vulnerable groups at risk of COVID-19. Therefore, public authorities have implemented a number of measures to address the needs of older adults and have increased their compliance with these measures. The older adults are expected to isolate if need be and comply with the preventive measures more easily than younger people (5). Frailty is a condition characterized by declining function across several homeostatic systems leading to increased vulnerability to stressors and the risk of adverse health outcomes. Thus, it is very likely that frailty, together with

Address for Correspondence: Burak Mete, Çukurova University Faculty of Medicine, Department of Public Health, Adana, Turkey Phone: +90 5447113164 E-mail: burakmete2008@gmail.com ORCID: orcid.org/0000-0002-0780-6176

Received: 05.11.2021 Accepted: 08.01.2022

Cite this article as: Mete B, Tanır F, Demirhindi H, Inaltekin A, Kanat C. Impact of the COVID-19 Pandemic on Frailty in Older Adults. Eur J Geriatr Gerontol 2022;4(2):79-84

๑Copyright 2022 by the Academic Geriatrics Society / European Journal of Geriatrics and Gerontology published by Galenos Publishing House. 
comorbidities, may have contributed to the high vulnerability to severe clinical manifestations and death from COVID-19 among older people. Although social isolation seems beneficial in preventing the spread of COVID-19 in older adults, it may have adverse psychological and physiological consequences. Frailty is a medical syndrome that increases the dependency on another individual in daily life and/or death risk and is characterized by reduced physical function, resistance, and strength involving several factors and causes. Frailty is regarded as a significant cause of morbidity and mortality in the older adults. Although frailty has several adverse outcomes, it can be prevented and remedied (6). This study aimed to examine the effects of the COVID-19 pandemic on frailty in a group of older adults at the end of the first year of the pandemic.

\section{Materials and Methods}

This cross-sectional study was conducted in Adana Province, Turkey, in 2021 in compliance with Helsinki Declaration. Approvals for the study were obtained from the Turkish Ministry of Health and Çukurova University's Ethical Committee (number: 112). The study population consisted of people aged 65 years and older. The minimum number of patients required for a sample with a type 1 error of 0.05 , type 2 error of 0.2 , and effect size of 0.222 was 345 (7). A total of 394 people were included. Convenience sampling was used as the sampling method. The older adults were contacted through primary care health centers (i.e., family medicine centers, community health centers) of Çukurova University Faculty of Medicine, Public Health Department's Practice and Research Areas in Adana City. Questionnaire forms were filled in during face-to-face interviews. Written informed consent was obtained from each participants. While filling out the questionnaires, infection control measures like physical distancing, mask wearing, and hand hygiene were implemented. The data collection form consisted of four parts and collected information on patients' socio-demographic data, FRAIL scale score, Scale of Adjustment to Measures in Respiratory Disease Outbreaks in the Older adults and fear of coronavirus scale score. The socio-demographic section collected information on age, sex, place of residence, education level, income, occupation, height, weight, comorbidities, smoking status, number of medicines taken daily, and the number of household members.

\section{Fear of COVID-19 scale}

This scale consists of a single dimension and comprises seven items. The scale did not contain any reverse items. The total score obtained from all the scale items reflects the level of COVID-19 fear experienced by the individual. The scores can range from seven to 35, with higher scores indicating a higher level of COVID-19 fear (8). The validity and reliability of the scale were assessed by Bakioğlu et al. (9) previously.

\section{Frail scale}

The FRAIL scale was used to determine a patient's frailty state. A validity-reliability study of the Turkish FRAIL scale was conducted by Muradi et al. in 2017. This scale has five components: Fatigue, resistance, ambulation, illness, and weight loss. Each component is scored as zero or one, with the total score ranging from zero to five. Scores are evaluated as 0, 1-2, and 3-5 as normal, prefrail, and frail, respectively (10). Frailty was evaluated in two sub-dimensions: The pre-pandemic period and pandemic period (the end of the first year). The individuals were asked to evaluate the sub-dimensions before and at the first year of the pandemic.

\section{Evaluation of the change in frailty}

The participants were asked to evaluate the sub-dimensions of the frailty scale once in the pre-pandemic period and during the sixth month after the start of the pandemic and the declaration of curfews (social isolation). The questions were as follows: Fatigue: "Before the start of the pandemic, how much time would you feel tired over a four-week period?" "After the start of the pandemic (now), how much of the time over the past four weeks did you feel tired?" Resistance: "Before the start of the pandemic, by yourself and not using aids, did you have any difficulty walking up ten steps without resting?" "After the start of the pandemic (now), by yourself and not using aids, did you have any difficulty walking up ten steps without resting?" Ambulation: "Before the start of the pandemic, by yourself and not using aids, did you have any difficulty walking several hundreds of meters?" "After the start of the pandemic (now), by yourself and not using aids, do you have any difficulty walking several hundreds of meters?" IIInesses: "Before the start of the pandemic, how many chronic illnesses did you have?" "After the start of the pandemic (now), how many chronic illnesses do you have?" Loss of weight: "Before the start of the pandemic, how much did you weigh with your clothes on but without shoes?" "After the start of the pandemic (now), how much do you weigh with your clothes on but without shoes?" A change of $>5 \%$ in weight was interpreted as frailty. The total frailty scale score was calculated by summing the scores for each sub-dimension for both the pre-pandemic period and at the sixth month of the pandemic. The difference between the pre-pandemic and post-pandemic scores (post-pandemic scores - pre-pandemic scores) yielded the final score change, with positive scores interpreted as an increase, negative scores as a decrease, and zero as unchanged.

\section{Scale of adjustment to measures in respiratory disease outbreaks in the elderly}

We recently developed the scale of adjustment to measures in respiratory disease outbreaks in the elderly. The scale contained 19 questions. An expert's council was utilized for the content 
validity of the scale, and only questions with a content validity index score of above 0.60 were included. The actual scale used was a five-point Likert scale consisting of 19 questions, with scores ranging from zero to four. The Kaiser-MeyerOlkin measure of sampling adequacy coefficient was 0.916, and Bartlett's test of sphericity had a $p$-value of $<0.001$ for suitability analyzes concerning the factor analysis for the data set. These data were confirmed as suitable for factor analysis. The Eigenvalues were 1 when obtaining the factors, with those above 1 accepted as factors. To determine the items to be included in the scale, an item analysis was performed, and itemtotal correlations were done. After these analyzes, the principal component analysis and Varimax rotation technique were used to determine the structure of the scale. In determining the scale items, the item-total correlation coefficients were required to be above 0.30 , factor load to be over 0.50 in the factor analysis, and for a single factor to have at least a difference of 0.1 from other factor structures for convergence assumption. Two items that did not meet these assumptions and two items were removed from the scale. The scale explains $62.36 \%$ of the total variance. The final version of the scale consisted of 17 questions and three factors. The first factor was "avoiding close physical contact" and consisted of six questions. The second factor was "hand-respiratory hygiene" and consisted of seven questions. The third factor was "self-isolation" and consisted of four questions. A minimum of zero and a maximum of 68 points can be obtained from the scale. An increase in the scores indicates more compliance with the measures. Cronbach's alpha method was used for the reliability analysis. The Cronbach's alpha coefficient for the final version of the scale was 0.915 (11).

\section{Statistics}

SPSS version 22 was used for the data analysis. Normal distribution was tested using the Kolmogorov-Smirnov test. A marginal homogeneity test, Wilcoxon test, and binary logistic regression analysis were performed to analyze the data. In the effect size analyses, Cohen's d values of greater than one indicated a very large effect, 0.8 a big effect, $0.2-0.5$ a moderate effect, and 0.2 a small effect. $p$-value $<0.05$ was considered statistically significant.

\section{Results}

The average age of the 394 older adult individuals who participated in our study was $70.38 \pm 5.68$ years (range: $65-$ 92 years). The socio-demographic characteristics of the study population are presented in Table 1. Of the older adults included in the study, 33\% were infected with COVID-19 and 86.8\% had inactivated severe acute respiratory syndrome-coronavirus-2 (SARS-CoV-2) vaccine. Moreover, 75.9\% of individuals had a chronic disease, with the most common being hypertension (Table 1).
When the change in frailty among older adults individuals between the pre-pandemic period and at the end of the first year of the pandemic was analyzed, it was found that the number of older adults individuals in the "normal" group decreased by $16.1 \%$. Consequently, this resulted in an increase of $2.7 \%$ in the prefrail group and $13.8 \%$ in the frail group $(p<0.001)$. The difference between the pre-pandemic and pandemic frailty scores was found to be statistically significant $(p<0.001)$. At the end of the first year of the pandemic, the effect of the pandemic on the frailty scores was found to be moderate, with Cohen's d of 0.403 (Table 2).

The logistic regression model set was found to be significant (Omnibus test $p<0.001$ ) for predicting the changes in the frailty group, including the presence of chronic diseases, the presence of COVID-19, the coronavirus fear score of the participants, vaccine status of participants and scale of adjustment to measures in respiratory disease outbreaks in the elderly. The accuracy of the model was found to be $70.6 \%$, with a Nagelkerke R square value of 0.099. It was found that the following variables contributed significantly to the model: Being infected with COVID-19, scale

\begin{tabular}{|l|l|}
\hline $\begin{array}{l}\text { Table 1. Socio-demographic characteristics and comorbidities } \\
\text { of the individuals }\end{array}$ \\
\hline Characteristics & n/\% \\
\hline Sex (male/female) & $196(49.7) / 198(50.3)$ \\
\hline Age (65-74/75-84/85 and above) & $311(78.9) / 72(18.3) / 11(2.8)$ \\
\hline $\begin{array}{l}\text { Education (illiterate/primary/ } \\
\text { elementary/high school/ } \\
\text { university) }\end{array}$ & $83(21.1) / 138(35.0) / 67$ \\
\hline $\begin{array}{l}\text { Income (2700 and } \\
\text { lower/2701-9300/9301 TL and } \\
\text { higher) }\end{array}$ & $142(36.4) / 224(57.4) / 24(6.2)$ \\
\hline Residential (city/town/village) & $189(48.3) / 144(36.8) / 58(14.8)$ \\
\hline Chronic diseases (yes/no) & $299(75.9) / 95(24.1)$ \\
\hline Hypertension & $187(47.6)$ \\
\hline Diabetes mellitus & $156(39.6)$ \\
\hline Cardiovascular diseases & $101(25.6)$ \\
\hline $\begin{array}{l}\text { Chronic obstructive pulmonary } \\
\text { disease }\end{array}$ & $67(17.0)$ \\
\hline Rheumatological diseases & $25(6.3)$ \\
\hline Malignancy & $5(1.3)$ \\
\hline Other & $49(12.4)$ \\
\hline $\begin{array}{l}\text { Number of daily drug } \\
\text { (0/1-3/4-7/8 and above) }\end{array}$ & $96(24.4) / 181(45.9) / 100$ \\
\hline Smoking (yes/no/quit) & 48 (12.2)/278 (70.6)/68 (17.3) \\
\hline CoVID-19 disease (yes/no) & $130(33.0) / 264(67.0)$ \\
\hline $\begin{array}{l}\text { Clinical severity (mild/moderate/ } \\
\text { serious) }\end{array}$ & $17(13.1) / 48(36.9) / 65(50.0)$ \\
\hline CoVID-19 vaccination (yes/no) & $342(86.8) / 52(13.2)$ \\
\hline Total & $394(100.0)$ \\
\hline CovID-19: Coronavirus disease-2019 & \\
\hline (17)
\end{tabular}


of adjustment to measures in respiratory disease outbreaks in the older adults and fear of COVID-19. The risk of frailty was found to be 2.04 times higher in individuals that tested positive for COVID-19. The 0.078 unit increase in the scores of the coronavirus fear scale increased the risk of frailty by 1.081 times. Every 0.031 unit increase in the scale reduced the risk of frailty by 1.031 times (Table 3 ).

When the scores obtained from the scale of adjustment to measures in respiratory disease outbreaks in the Older adults were compared according to the status of having COVID-19 infection, it was found that there was a statistically significant difference between the total score obtained from the scale and the scores obtained from the hand-respiratory hygiene factor. It was found that the scores obtained from the sum of the scale and the hand-respiratory hygiene sub-factor were statistically lower in people who had COVID-19, that is, their compliance with non-pharmacological measures was less (Table 4).

\section{Discussion}

The COVID-19 pandemic moved across the globe at an unprecedented speed and has a number of health and socioeconomic effects (1). The older adults are one of the risk groups most affected by the COVID-19 pandemic, with it having many direct and indirect effects on the elderly population. Its direct effects are the negative effects of the disease itself (postCOVID-19 syndrome). To mitigate these effects, patients who recover should be examined for post-COVID-19 manifestations and followed up for a long time (12). There is also a need for studies on indirect effects, which may become a problem in the future. In a study by Heckman et al. (13), it was argued that the measures applied to the elderly during the pandemic period would indirectly increase the strain on hospitals. It can be expected that the number of frail elderly will increase due to both the measures implemented to stop the spread of COVID-19 and the disease itself.

\begin{tabular}{|c|c|c|c|c|}
\hline Frailty groups & \begin{tabular}{|l|} 
Before \\
the pandemic \\
$n(\%)$
\end{tabular} & $\begin{array}{l}\text { At } 1^{\text {st }} \text { year } \\
\text { of the pandemic } \\
n(\%)\end{array}$ & $\begin{array}{l}\text { Change } \\
(\%)\end{array}$ & $\mathbf{p}$ \\
\hline Normal & 191 (49.2) & $129(33.1)$ & -16.1 & \\
\hline Prefrail & $148(38.1)$ & $159(40.8)$ & 2.7 & $<0.001$ \\
\hline \multirow[t]{2}{*}{ Frail } & 49 (12.4) & $102(26.2)$ & 13.8 & \\
\hline & $\bar{x} \pm S D$ & $\bar{x} \pm S D$ & Cohen's d & $p$ \\
\hline Frailty score & $0.98 \pm 1.18$ & $1.50 \pm 1.39$ & 0.403 & $<0.001$ \\
\hline
\end{tabular}

Table 3. Logistic regression model for predicting the impact of the COVID-19 pandemic on frailty

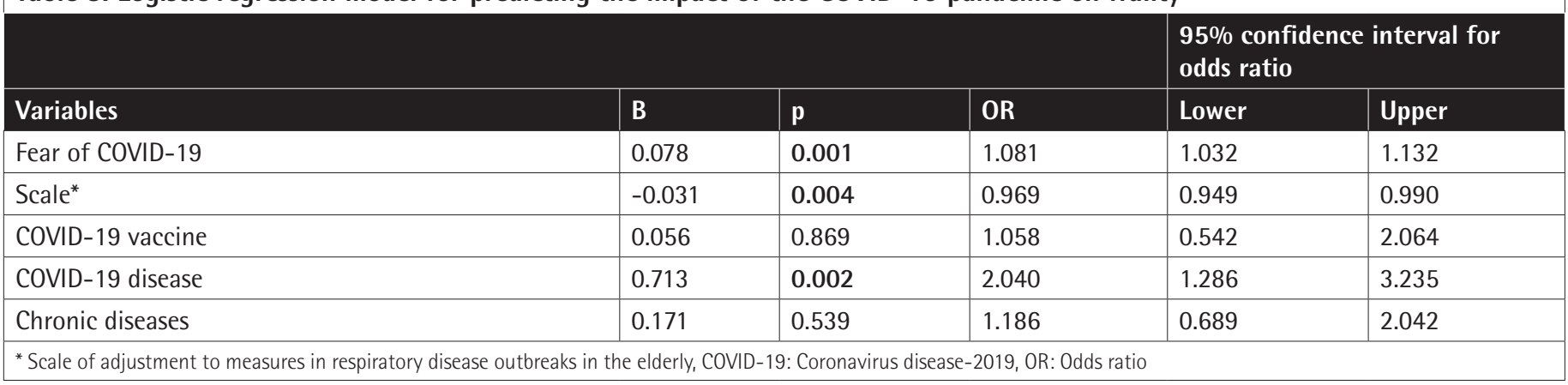

Table 4. Comparison of scores from the scale according to COVID-19 disease

\begin{tabular}{|c|c|c|c|c|c|}
\hline \multirow[b]{3}{*}{ Scale score } & \multicolumn{4}{|c|}{ COVID-19 diagnosis } & \multirow[b]{3}{*}{$\mathbf{p}$} \\
\hline & \multicolumn{2}{|l|}{ No } & \multicolumn{2}{|l|}{ Yes } & \\
\hline & $\bar{X} \pm S D$ & Median & $\bar{X} \pm S D$ & Median & \\
\hline Factor 1 & $15.84 \pm 5.75$ & 17.0 & $15.66 \pm 4.59$ & 16.0 & 0.185 \\
\hline Factor 2 & $21.17 \pm 5.34$ & 21.0 & $19.79 \pm 5.58$ & 19.0 & 0.009 \\
\hline
\end{tabular}


A study by Vetrano et al. (14) argued that social isolation measures taken to reduce viral transmission might have significant adverse effects on the elderly living with multiple morbidities. Social isolation is likely to affect formal and informal care, leading to loneliness, depression, anxiety, accelerated functional and cognitive decline, falls, and fractures (15). A study by Mete et al. (7) found that frailty, falls at home, and cognitive decline increased among elderly individuals during heightened periods of social isolation. It is also possible that the burden of care of these older adults might increase with mild complaints worsening enough to require hospitalization. When hospitalized, these individuals will be more isolated and at higher risk for functional decline, resulting in longer hospital stays and the need for post-discharge home care, rehabilitation, or further hospitalization (15). Increased frailty among the elderly may increase these problems and increase the indirect burden in the post-pandemic period. According to the results of our study that evaluated a group of older adults at the end of the first year of the pandemic, the rate of prefrail and frail elderly increased by $2.7 \%$ and $13.8 \%$, respectively. The impact of the pandemic on frailty in this elderly population was found to be moderate. According to a study we conducted in the first six months of the pandemic in the same region, the percentage of pre-frailty and frailty among the elderly increased by $4.4 \%$ and $6.6 \%$, respectively, and the impact of the pandemic on frailty was small (7). It is seen that the rate of frail older adults and the magnitude of the effect of the pandemic on frailty have increased in the second six-month period. The increase in the number of older adults with COVID-19 may partly explain this increase in frailty. We found that the risk of frailty increased two-fold in those older adults who had tested positive for COVID-19, while the fear of getting sick increased the risk of frailty by 1.081 -fold. In addition, it has been observed that increasing compliance with the measures taken reduces the risk of frailty. In particular, compliance with hand-respiratory hygiene (hand disinfection and wearing a mask) is lower in people who have had the disease. There is evidence that the implementation of universal mask reduces the spread of COVID-19. In a study conducted on healthcare workers in a hospital, after a mandatory mask application, new infections among health workers (HCWs) with direct or indirect patient contact were increasing exponentially, from 0\% to 21.3\% (a mean increase of $1.16 \%$ per day). However, after the universal masking policy was in place, the proportion of symptomatic HCWs with positive test results steadily declined, from $14.7 \%$ to $11.5 \%$ (a mean decrease of $0.49 \%$ per day). Although not a randomized clinical trial, this study provides critically important data to emphasize that masking helps prevent transmission of SARS-CoV-2 (16).

Pre-COVID studies reported a difference between frailty categories in terms of clinical outcomes (17). Acute disease is less tolerated among the more frail older adults. The severity of the disease and the degree of frailty are important for clinical outcomes (18). Frailty is not synonymous with end-oflife. In a non-COVID-19 related study of 15,613 patients aged $\geq 80$ years in intensive care units across Australia, those with a CFS $\geq 5$ had significantly poorer health outcomes than agematched peers who were less frail. However, the prevalence of in-hospital mortality (17.6\% versus $8.2 \%)$ and new discharges to residential aged care facilities ( $4.9 \%$ versus $2.8 \%$ ) suggest the majority of frail patients do survive and return home to the community (19). It was found in a systematic review conducted by Maltase et al. (20) during the COVID-19 period that intensive care hospitalization and mortality were higher among frail older adults compared to healthy older adults. Moreover, in a cohort study by Aw et al. (21) that classified 674 patients in terms of frailty, the risk of mortality was found to be 2.13 times higher in patients with high frailty during an average follow-up period of 34.3 days. The COVID-19 pandemic increases both the frailty and risk of negative outcomes in frail elderly.

\section{Study Limitations and Conclusion}

This study has several limitations in that it was conducted in a single region and used non-probability sampling.

In our study, both the fear of getting sick and the disease itself was found to increase the risk of frailty in older adults at the end of the first year of the COVID-19 pandemic. Testing positive for COVID-19 was found to be the factor that increased the risk of frailty the most. It has been found that compliance with the measures implemented to combat the pandemic protected from frailty. We assert that the risk of frailty will be reduced by ensuring that all older adults have at least two doses of a COVID-19 vaccine and comply with the recommended handrespiratory hygiene measures. Otherwise, indirect effects of the pandemic on older adults, such as social isolation, will create a significant burden in the post-pandemic period.

\section{Ethics}

Ethics Committee Approval: This cross-sectional study was conducted in Adana Province, Turkey, in 2021 in compliance with Helsinki Declaration. Approvals for the study were obtained from the Turkish Ministry of Health and Çukurova University's Ethical Committee (number: 112).

Informed Consent: Written informed consent was obtained from each participants.

Peer-review: Externally peer-reviewed.

\section{Authorship Contributions}

Surgical and Medical Practices: B.M., Concept: B.M., A.I., C.K., Design: B.M., F.T., A.I., C.K., Data Collection or Processing: B.M., 
H.D., A.I., C.K., Analysis or Interpretation: B.M., H.D., Literature Search: B.M., F.T., Writing: B.M., H.D.

Conflict of Interest: No conflict of interest was declared by the authors.

Financial Disclosure: The authors declared that this study received no financial support.

\section{References}

1. Chen $Y$, Klein SL, Garibaldi BT, Li H, Wu C, Osevala NM, Li T, Margolick $J B$, Pawelec G, Leng SX. Aging in COVID-19: Vulnerability, immunity and intervention. Ageing Res Rev 2021;65:101205.

2. Wu Z, McGoogan JM. Characteristics of and Important Lessons From the Coronavirus Disease 2019 (COVID-19) Outbreak in China: Summary of a Report of 72314 Cases From the Chinese Center for Disease Control and Prevention. JAMA 2020;323:1239-1242.

3. Zhu N, Zhang D, Wang W, Li X, Yang B, Song J, Zhao X, Huang B, Shi W, Lu R, Niu P, Zhan F, Ma X, Wang D, Xu W, Wu G, Gao GF, Phil D, Tan W; China Novel Coronavirus Investigating and Research Team. A Novel Coronavirus from Patients with Pneumonia in China, 2019. N Engl J Med 2020;382:727733.

4. Yip W, Fu H, Chen AT, Zhai T, Jian W, Xu R, Pan J, Hu M, Zhou Z, Chen Q, Mao W, Sun $\mathrm{Q}$, Chen W. 10 years of health-care reform in China: progress and gaps in Universal Health Coverage. Lancet 2019;394:1192-1204.

5. Daoust JF. Elderly people and responses to COVID-19 in 27 Countries. PLoS One 2020;15:e0235590.

6. Tribess $\mathrm{S}$, de Oliveira RJ. [Biological fragility syndrome in the elderly: systematic review]. Rev Salud Publica (Bogota) 2011;13:853-864.

7. Mete B, Tanır F, Kanat C. The Effect of Fear of COVID-19 and Social Isolation on the Fragility in the Elderly. Turkish Journal of Geriatrics 2021;24:23-31.

8. Ahorsu DK, Lin CY, Imani V, Saffari M, Griffiths MD, Pakpour AH. The Fear of COVID-19 Scale: Development and Initial Validation. Int J Ment Health Addict 2020;27:1-9.

9. Bakioğlu F, Korkmaz O, Ercan H. Fear of COVID-19 and Positivity: Mediating Role of Intolerance of Uncertainty, Depression, Anxiety, and Stress. Int J Ment Health Addict 2021;19:2369-2382.
10. Morley JE, Malmstrom TK, Miller DK. A simple frailty questionnaire (FRAIL) predicts outcomes in middle aged African Americans. J Nutr Health Aging 2012;16:601-608.

11. Mete $B$, Tanır $F$, İnaltekin $A$. Yaşlılarda solunum yolu salgınlarında önlemlere uyum ölçeği-geçerlilik ve güvenirlik çalışması. Sağ Aka Derg 2022;9:91-98.

12. Kamal $M, A b o$ Omirah $M$, Hussein $A$, Saeed $H$. Assessment and characterisation of post-COVID-19 manifestations. Int J Clin Pract 2021;75:e13746.

13. Heckman GA, Saari M, McArthur C, Wellens NIH, Hirdes JP. COVID-19 outbreak measures may indirectly lead to greater burden on hospitals. CMAJ 2020;192:E384.

14. Vetrano DL, Palmer $K$, Marengoni $A$, Marzetti $E$, Lattanzio $F$, RollerWirnsberger R, Samaniego LL, Rodriguez-Manas L, Bernabei R, Onder G; Joint Action ADVANTAGE WP4 Group. Frailty and Multimorbidity: A Systematic Review and Meta-analysis. J Gerontol A Biol Sci Med Sci 2019;74:659-666.

15. Wodchis WP, Austin PC, Henry DA. A 3-year study of high-cost users of health care. CMAJ 2016;188:182-188.

16. Brooks JT, Butler JC, Redfield RR. Universal Masking to Prevent SARS-CoV-2 Transmission-The Time Is Now. JAMA 2020;324:635-637.

17. Rockwood $K$, Song $X$, MacKnight $C$, Bergman H, Hogan DB, McDowell I, Mitnitski A. A global clinical measure of fitness and frailty in elderly people. CMAJ 2005;173:489-495.

18. Romero-Ortuno $R$, Wallis $S$, Biram $R$, Keevil V. Clinical frailty adds to acute illness severity in predicting mortality in hospitalized elderly: An observational study. Eur J Intern Med 2016;35:24-34.

19. Darvall JN, Bellomo R, Paul E, Subramaniam A, Santamaria JD, Bagshaw $S M$, Rai $S$, Hubbard RE, Pilcher D. Frailty in very old critically ill patients in Australia and New Zealand: a population-based cohort study. Med J Aust 2019;211:318-323.

20. Maltese G, Corsonello A, Di Rosa M, Soraci L, Vitale C, Corica F, Lattanzio F. Frailty and COVID-19: A Systematic Scoping Review. J Clin Med 2020;9:2106.

21. Aw D, Woodrow L, Ogliari G, Harwood R. Association of frailty with mortality in older inpatients with Covid-19: a cohort study. Age Ageing 2020;49:915922. 